\title{
Integrating a 92-Gene Expression Analysis for the Management of Neuroendocrine Tumors of Unknown Primary
}

\author{
Aman Chauhan ${ }^{1 *}$, Zainab Farooqui ${ }^{2}$, Scott R Silva ${ }^{3}$, Le Aundra Murray ${ }^{4}$, Kurt \\ B Hodges $^{5}$, Qian Yu ${ }^{6}$, Zin W Myint ${ }^{1}$, Arumugam K Raajesekar ${ }^{1}$, Heidi Weiss ${ }^{7,8,9}$, \\ Susanne Arnold ${ }^{1,9}$, B Mark Evers ${ }^{8}$, Lowell Anthony,
}

\begin{abstract}
Background: Neuroendocrine tumors (NETs) are rare tumors that can originate from any part of the body. Often, imaging or exploratory surgery can assist in the identification of the tumor primary site, which is critical to the management of the disease. Neuroendocrine tumors (NETs) of unknown primary constitute approximately 10-15\% of all NETs. Determining the original site of the tumor is critical to providing appropriate and effective treatment. Methods: We performed a retrospective review of neuroendocrine tumors at our institution between 2012 and 2016 using a 92-gene cancer ID analysis. Results: 56 patients with NETs of unknown primary were identified. Samples for 38 of the 56 underwent the 92 -gene cancer ID analysis. The primary site of the tumor was identified with $>95 \%$ certainty in 35 of the 38 patients. Conclusion: The 92 -gene cancer ID analysis identified a primary site in $92 \%$ of our NETs study cohort that previously had been unknown. The results have direct implications on management of patients with regard to FDA-approved treatment options.
\end{abstract}

Keywords: Neuroendocrine tumor- tumor of unknown primary- 92-gene cancer ID assay

Asian Pac J Cancer Prev, 20 (1), 113-116

\section{Introduction}

Neuroendocrine tumors (NETs) are rare tumors that can originate from any part of the body. The gastrointestinal tract and lung are the most common sites of origin, but NETs originating from ovary, breast, cervix as well as other sites have been reported in the literature (Zuetenhorst and Taal, 2005). Often, imaging or exploratory surgery can assist in the identification of the tumor primary site, which is critical to the management of the disease. A variety of therapies, including targeted agents, are FDA-approved for specific NETs subtypes: sunitinib is approved for management of pancreatic neuroendocrine tumors, everolimus is approved for gastroenteropancreatic and bronchial NETs, immune checkpoint inhibitors are active in Merkel cell carcinoma, while metaiodobenzylguanidine is standard of care for pheochromocytoma. Hence, knowledge of the primary site is immensely important for directing therapy. Despite our best imaging and interventional diagnostics, we are unable to determine the primary site for all NET tumors. These NETs of unknown primary are a substantial subset, 10$15 \%$, of all neuroendocrine tumors (Zuetenhorst and Taal,
2005). CancerTYPE ID (Biotheranostics, San Diego, CA), is a 92-gene real-time quantitative reverse transcriptase polymerase chain reaction (RT-PCR) gene expression assay, performed in Biotheranostic's CLIA-certified laboratory that can be used to determine the origin of NETs of unknown primary, thereby guiding physicians in therapy selection. Here, we review the history of this technology and present our single-center experience using CancerTYPE ID in management of NETs of unknown primary.

\section{Materials and Methods}

\section{Methods}

Markey Cancer Center is a referral center for neuroendocrine tumors nationwide. Patients at the University of Kentucky who were diagnosed with neuroendocrine tumor of unknown primary over a five-year period (from 2012 to 2016) were identified from a Markey Cancer Center's database. A subset of these underwent the 92-gene RT-PCR analysis and electronic medical records of these patients were retrospectively reviewed. Studies were performed in accordance with and

${ }^{1}$ Department of Internal Medicine, Division of Medical Oncology, ${ }^{5}$ Department of Pathology and Laboratory Medicine, ${ }^{6}$ College of Medicine, ${ }^{7}$ Department of Biostatistics, College of Public Health, ${ }^{8}$ Department of Surgery, ${ }^{9}$ Markey Cancer Center, University of Kentucky, ${ }^{4}$ University of Kentucky, Lexington, ${ }^{2}$ University of Kentucky, College of Osteopathic Medicine, Pikeville, KY, ${ }^{3}$ Department of Radiation Oncology, Stritch School of Medicine Loyola University Chicago, Maywood, IL, United States. *For Correspondence: amanchauhan@uky.edu 
the approval of the University Institutional Review Board.

\section{Results}

We identified 56 patients with neuroendocrine tumor of unknown primary. The median age of patients was 61 years; $50 \%$ were male. $92 \%$ patients were Caucasians and rest were African Americans. Almost entire study cohort belonged to Appalachian region of Kentucky and surrounding states. The 92-gene expression analysis was performed in 38 of the 56 patients. Some patients could not get molecular testing doe due to insurance coverage/ financial constraints. The primary site of the tumor was identified with $>95 \%$ certainty in 35 of the 38 patients. The test reported gastrointestinal NETs as the primary site in 14 patients, pancreatic as the primary site in 10 patients, bronchial carcinoid in five, large cell neuroendocrine carcinoid in three, Merkel cell carcinoma in two, and pheochromocytoma in one patient (Figure 1).

\section{Discussion}

Cancer diagnostics have experienced rapid advancements, but despite improvements in diagnostic accuracy, a small percentage of carcinomas remain uncategorized as to site of origin and are classified as cancers of unknown primary (CUP) when metastases are diagnosed. The challenge of correctly identifying the site of the primary tumor more often presents in cases with poorly differentiated tumors, an atypical metastatic site, or multiple probable diagnoses $(\mathrm{Wu}$ et al., 2012). The first step in successful management of a cancer involves obtaining an accurate histopathological diagnosis, which is why clinicians and pathologists often request extensive and costly measures to determine the primary site through imaging techniques, serum tests, immunohistochemical (IHC) panels and morphological feature analysis (van Laar et al., 2009, Pillai et al., 2011). Though these diagnostic tools are useful in the evaluation process, recent blinded studies show an accuracy rate of $66-69 \%$ for the identification of primary sites through IHC staining-the most commonly used ancillary study for evaluation of metastatic CUP (Ma et al., 2006, Pillai et al., 2011, Weiss et al., 2013). The lack of sensitivity and specificity necessary for definitive identification of primary sites using current clinco-pathological tools validates the crucial need for new, revolutionary clinical diagnostic approaches. Molecular classification through this 92-gene expression analysis has provided promising results in accurately identifying the tumor primary site in difficult metastatic carcinomas (Ma et al., 2006, Wu et al., 2012).

Many studies support molecular gene expression profiling as an accurate classifier of common tumor types (Ramaswamy et al., 2001, Bloom et al., 2004, Tothill et al., 2005, Ma et al., 2006). Many differentiated tumors maintain commonalities on a molecular level, and these findings were the premise for constructing a universal gene expression reference database that could analyze a variety of cancer types and be utilized as a diagnostic tool. The resulting success rate for molecular profiling, using RT-PCR technology, ranged from $75 \%$ to $89 \%$, a definite improvement from the 66-69\% achieved with IHC (Wu et al., 2012). Based on these promising results aiding cancer classification, modifications were initiated, with the construction of a 22,000-gene oligonucleotide microarray to develop a comprehensive reference database from 578 tumors. This microarray represented a wide-spectrum of tissue origins and histologic subtypes. The assay was designed and PCR data were analyzed and provided accuracy for formalin fixed/paraffin embedded (FFPE) and frozen samples) (Ma et al., 2006). This led to the development of the TaqMan RT-PCR 92-gene test, consisting of 87 tumor-related genes and 5 reference genes, designed for high success in cancer classification, which included genes encoding transcription factors and cell surface receptors (Ma et al., 2006, Erlander et al., 2011). A recent reassessment expanded the database to 30 major cancer types and 54 histological subtypes (Erlander et al., 2011). Analysis of the 92-gene test allowed performance comparisons between primary and metastasis tumor sites, tumor grades, and previous microarray platform studies (Erlander et al., 2011, Wu et al., 2012). No performance loss or significant difference between primary and metastatic sites was found and no statistical significance among tumor grades could be identified ( $\mathrm{Wu}$ et al., 2012) and was proven accurate with smaller tissue samples, irrespective of biopsy site (Brachtel et al., 2016). The 92-gene cancer ID analysis accurately determined the tumor site origin of $96.2 \%$ of cases compared to IHC,

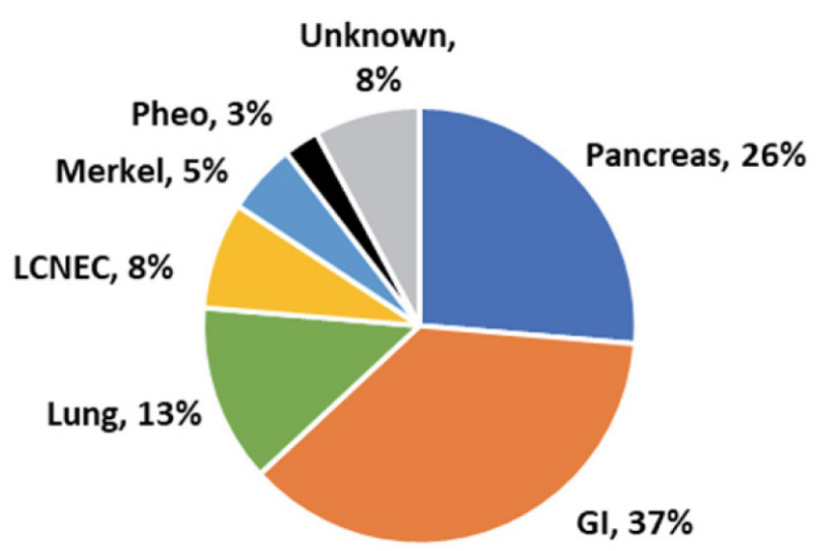

Figure 1. Distribution of NET of Unknown Primary at Markey Cancer Center (2012-2016) Based on the 92-Gene Expression Assay 
which correctly indicated the primary site in $85.2 \%$ of cases (Maxwell et al., 2014). An increase in survival, improved life quality and a decrease in misdiagnoses and decreased cost were positive attributes identified from a patient's perspective. (Bentley et al., 2014).

There is considerable evidence that the 92 -gene cancer ID analysis is an efficient and accurate diagnostic tool that should be adopted in standardized clinical evaluations of patients with metastatic cancers with an unknown primary sites for all disease types. Most patients who receive a diagnosis of CUP undergo empiric therapies that provide low to moderate benefits and poor survival rates (Hainsworth et al., 2012). Investigators used the 92-gene cancer ID assessment to identify the tissue origin of 289 patients with CUP and consequently treated 194 patients with gene-directed, site-specific therapy (Hainsworth et al., 2013). Median survival was 12.5 months with the CancerTYPE ID-predicted chemo-sensitive tumor cohort vs 9.1 months for the historical control arm (Hainsworth et al., 2013). The CancerTYPE ID predicted resistant tumors and survival was well-predicted. An accurate classification of carcinomas would reap increased benefits from site-specific therapies, ultimately improving survival and overall treatment of patients with these diagnoses.

Neuroendocrine tumors are rare, slow-growing neoplasms that can originate from a wide variety of tissues and organ sites, including the pancreas, ovaries, breast, and thymus. However, these tumors most commonly stem from the gastrointestinal tract (65\%) and the lungs (25\%) (Zuetenhorst and Taal, 2005). Based in part on this widely diverse tissue of origin, NETs derive from cells that produce neurotransmitters, neuropeptide hormones, secretory granules, and active amines (Barakat et al., 2004). Expression of these cellular products in NETs means that patients can experience diverse symptoms and require a multidisciplinary approach for management. A complicating factor in the management of NETS is the need to recognize the distinction between low/ intermediate-grade well-differentiated carcinomas and high-grade poorly-differentiated carcinomas because the most efficacious therapies for these subgroups differ considerably.

NETs with a known primary are fairly manageable thanks to a variety of FDA-approved site-specific regimens. However, it is important for physicians to accurately and efficiently diagnose neuroendocrine tumors of unknown primary based on a site-specific origin.(Sherman et al., 2014) This identification is often difficult. Some primary sites, such as the small bowel (SBNET) and pancreatic neuroendocrine tumors (PNET), are indistinguishable on $\mathrm{CT}$ scan, routine hematoxylin and eosin stains, or other diagnostic techniques due to similarities in morphology and phenotype within this tumor type (Maxwell et al., 2014). Importantly, medical treatment for pancreatic NETs currently includes peptide receptor radio therapy, sunitinib and everolimus, whereas for thoracic NETs treatment options are more limited (everolimus)

Therefore, despite extensive diagnostics, approximately $10 \%$ of metastatic NET cases continue to be NET of unknown primary at the time of diagnosis
(Zuetenhorst and Taal, 2005). Although a variety of biomarkers, IHC or other staining techniques have been presented as a means for distinguishing NETs initiated from the GI, pancreas, and lung (Kerr et al., 2014, Maxwell et al., 2014), these methodologies lack specificity.

The 92-gene cancer ID analysis, one of a group of gene expression molecular classifiers (GEMC), that has shown promise for clinical utility in not only carcinoma of unknown primary but also in gastrointestinal NETs. With respect to analysis of NETs in particular, an algorithm was constructed and validated for the 92-gene cancer ID analysis that correctly identified PNETs from SBNETs based on four molecular genes; this analysis accurately identified the primary sites of 52 of 56 liver metastases with $92.9 \%$ sensitivity (Sherman et al., 2014).

Ongoing studies of gene expression cancer classifier assessments for application of origin-site predictions for NETs are not prevalent, but are on the rise. In 2011, a blinded, multi-facility study validated the diagnostic precision of the 92-gene cancer analysis using 790 cases comprised of 28 cancer types and more than 50 cancer subtypes (Kerr et al., 2012). Two years later, a second blinded study by the same group (Kerr et al., 2014) established the clinical feasibility and value of utilizing the 92-gene cancer analysis to distinguish subtypes of neuroendocrine tumors with unknown primary sites. 75 NET patients were studied. 44 samples were from a metastatic site and 31 were from the primary tumor. Two gastrointestinal pathologists confirmed the diagnoses and grades (well-differentiated tumors, grades 1 and 2, and poorly-differentiated and Merkel cell tumors, grade 3). The raw data from the 92-gene analysis was compared to the reference database; the rate of concordance for poorly-differentiated and well-differentiated NET were $87 \%$ and $97 \%$, respectively. Four case predictions showed discordance in the subtype, and these were of poorlydifferentiated tumors, but the analysis accurately predict the NET tumor type. The sensitivities for accuracy were $99 \%$ for neuroendocrine tumor classification and $95 \%$ for NET subtype for primary site classification. These results validate the accuracy of the 92-gene molecular cancer classifier for diagnosing subtypes of NETs.

In our study cohort, we were able to utilize the 92-gene cancer ID analysis in clinical management of patients and were able to treat with appropriate FDA approved agents. With an increase in FDA-approved treatments tailored for organ- specific sites of NETs, the clinical application of 92-gene cancer ID assay for differentiating subtypes of neuroendocrine carcinomas has the potential to improve outcomes. In our study at the University of Kentucky Markey Cancer Center, the 92-gene cancer ID analysis identified the NET primary site for tumors that was otherwise unknown for a majority (94.7\%) of our study cohort. The results had direct implications on management of patients with regard to FDA-approved treatment options.

\section{Declarations}

Ethics approval and consent to participate: Ethics approval was obtained from University of Kentucky IRB: Protocol Number 17-0371-P2H. Need for informed

Asian Pacific Journal of Cancer Prevention, Vol 20 
consent was waived.

\section{Author contributions}

All authors actively participated in the data collection, analysis interpretation and writing of this manuscript. All authors read and approved the final manuscript.

\section{Conflict of Interest \\ No Conflicts to disclose.}

\section{Acknowledgments}

This research was supported by the Biostatistics and Bioinformatics Shared Resource of the University of Kentucky Markey Cancer Center (P30CA177558). The authors also wish to thank Donna Gilbreath and Cathy Anthony in the Markey Cancer Center's Research Communications Office for assistance in preparing this manuscript.

\section{References}

Barakat MT, Meeran K, Bloom SR (2004). Neuroendocrine tumours. Endocr Relat Cancer, 11, 1-18.

Bentley TG, Schroeder BE, Schnabel CA, et al. (2014). Cost effectiveness of a 92-gene assay for the diagnosis of metastatic cancer. J Med Econ, 17, 527-37.

Bloom G, Yang IV, Boulware D, et al (2004). Multi-platform, multi-site, microarray-based human tumor classification. Am J Pathol, 164, 9-16.

Brachtel EF, Operana TN, Sullivan PS, et al (2016). Molecular classification of cancer with the 92-gene assay in cytology and limited tissue samples. Oncotarget, 7, 27220-31.

Erlander MG, Ma XJ, Kesty NC, et al. (2011). Performance and clinical evaluation of the 92-gene real-time pcr assay for tumor classification. J Mol Diagn, 13, 493-503.

Hainsworth JD, Rubin MS, Spigel DR, et al (2013). Molecular gene expression profiling to predict the tissue of origin and direct site-specific therapy in patients with carcinoma of unknown primary site: A prospective trial of the sarah cannon research institute. J Clin Oncol, 31, 217-23.

Hainsworth JD, Schnabel CA, Erlander MG, et al (2012). A retrospective study of treatment outcomes in patients with carcinoma of unknown primary site and a colorectal cancer molecular profile. Clin Colorectal Cancer, 11, 112-8.

Kerr SE, Schnabel CA, Sullivan PS, et al (2014). A 92-gene cancer classifier predicts the site of origin for neuroendocrine tumors. Mod Pathol, 27, 44-54.

Kerr SE, Schnabel CA, Sullivan PS, et al (2012). Multisite validation study to determine performance characteristics of a 92-gene molecular cancer classifier. Clin Cancer Res, 18, 3952-60.

Ma XJ, Patel R, Wang X, et al (2006). Molecular classification of human cancers using a 92-gene real-time quantitative polymerase chain reaction assay. Arch Pathol Lab Med, 130, 465-73.

Maxwell JE, Sherman SK, Stashek KM, et al (2014). A practical method to determine the site of unknown primary in metastatic neuroendocrine tumors. Surgery, 156, 1359-65; discussion 65-6.

Pillai R, Deeter R, Rigl CT, et al (2011). Validation and reproducibility of a microarray-based gene expression test for tumor identification in formalin-fixed, paraffin-embedded specimens. J Mol Diagn, 13, 48-56.

Ramaswamy S, Tamayo P, Rifkin R, et al (2001). Multiclass cancer diagnosis using tumor gene expression signatures. Proc Natl Acad Sci U S A, 98, 15149-54.

Sherman SK, Maxwell JE, Carr JC, et al (2014). Gene expression accurately distinguishes liver metastases of small bowel and pancreas neuroendocrine tumors. Clin Exp Metastasis, 31, 935-44.

Tothill RW, Kowalczyk A, Rischin D, et al (2005). An expression-based site of origin diagnostic method designed for clinical application to cancer of unknown origin. Cancer Res, 65, 4031-40.

van Laar RK, Ma XJ, de Jong D, et al (2009). Implementation of a novel microarray-based diagnostic test for cancer of unknown primary. Int J Cancer, 125, 1390-7.

Weiss LM, Chu P, Schroeder BE, et al (2013). Blinded comparator study of immunohistochemical analysis versus a 92-gene cancer classifier in the diagnosis of the primary site in metastatic tumors. J Mol Diagn, 15, 263-9.

Wu F, Huang D, Wang L, et al (2012). 92-gene molecular profiling in identification of cancer origin: A retrospective study in chinese population and performance within different subgroups. PLoS One, 7, e39320.

Zuetenhorst JM, Taal BG (2005). Metastatic carcinoid tumors: A clinical review. Oncologist, 10, 123-31.

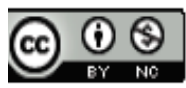

This work is licensed under a Creative Commons AttributionNon Commercial 4.0 International License. 